\title{
Relación entre conocimientos, actitudes y prácticas en la prevención de los riesgos ergonómicos de los profesionales de enfermería de la Clínica Good Hope, Lima, 2016
}

\author{
Relationship between knowledge, attitudes and practices in the prevention of the ergonomic risks of \\ nursing professionals at the Good Hope Clinic, Lima, 2016
}

\author{
Paulina Tipantuña Malte ${ }^{1 *}$, Wendy Reyes Miguel², Ángela Paredes Aguirre ${ }^{3}$
}

\begin{abstract}
RESUMEN
Objetivo: Determinar la relación entre conocimientos, actitudes y prácticas en la prevención de riesgos ergonómicos de los profesionales de Enfermería de la Clínica Good Hope. Materiales y Métodos: Diseño correlacional de corte transversal. La muestra fue no probabilística y por conveniencia, conformada por 130 profesionales de Enfermería. Los instrumentos utilizados para la recolección de datos fueron de elaboración propia contando con una validez de 1 a través de la V de Aiken y una confiabilidad de 0,890 con Alfa de Cronbach. Resultados: A través de la prueba chi cuadrado se encontró que existe relación entre los conocimientos y prácticas $(\mathrm{p}=0.025)$ en la prevención de riesgos ergonómicos. Sin embargo no existe relación entre los conocimientos y actitudes ( $\mathrm{p}=$ 0.076) y entre las actitudes y prácticas $(\mathrm{p}=0.133)$. Conclusiones: Se encontró relación entre los conocimientos y prácticas hacia la prevención de riesgos ergonómicos.
\end{abstract}

Palabras claves: Riesgos ergonómicos, enfermería, actitud, conocimientos y prácticas.

\begin{abstract}
Objective: To determine the relationship between knowledge, attitudes and practices in the prevention of ergonomic risks of nursing professionals at the Good Hope Clinic. Materials and Methods: Cross-sectional correlational design. The sample was non-probabilistic and by convenience, conformed by 130 professionals of Nursing. The instruments used for the data collection were of own elaboration counting with a validity of 1 through V of Aiken and a reliability of 0,890 with Alpha of Cronbach. Results: The chi-square test showed that there is a relationship between knowledge and practices $(\mathrm{p}=0.025)$ in the prevention of ergonomic risks. However, there is no relationship between knowledge and attitudes $(p=0.076)$ and between attitudes and practices $(\mathrm{p}=0.133)$. Conclusions: A relationship was found between knowledge and practices towards the prevention of ergonomic risks.
\end{abstract}

Keywords: Ergonomic risks, nursing, attitude, knowledge and practices.

\footnotetext{
${ }^{I}$ Licenciada en Enfermería, EP Enfermería, Facultad de Ciencias de la Salud.

${ }^{2}$ Licenciada en Enfermería, EP Enfermería, Universidad Peruana Unión, Lima Perú.

${ }^{3}$ Directora de la Unidad de Posgrado, Escuela de Posgrado, Universidad Peruana Unión, Lima, Perú.
} 


\section{INTRODUCCIÓN}

Creus (2012), definió a la ergonomía como una ciencia que estudia la relación entre el hombre y su ambiente de trabajo. Por otro lado Melorose, Perroy, y Careas (2015), mencionaron que los riesgos ergonómicos son probabilidades de sufrir un evento adverso e indeseado como accidente o enfermedad en el trabajo, condicionado por características que son capaces de generar una serie de trastornos o lesiones.

La OMS (2014), sostuvo que en diversos países, más de la mitad de los trabajadores carecen de protección y no existen mecanismos de aplicación de las normas sobre salud y seguridad ocupacional. Los riesgos con mayor carga de morbilidad fueron: traumatismos, ruidos, y riesgos ergonómicos.

En tanto la Organización Panamericana de la Salud (2001), refirió que es inaceptable que las personas pierdan la salud o incluso sus vidas por la rutina de su actividad laboral. Los accidentes y enfermedades ocupaciones significan sufrimiento humano para el trabajador y su familia, el esfuerzo físico es parte esencial de algunas actividades laborales, no sólo es un componente de los trabajos. A veces el mantenimiento de una misma postura durante ocho horas puede ser la causa de lesiones corporales.

Por otro lado, Sezgin y Esin (2015) en un estudio aplicado a Enfermeras en Turquía encontró que existe una alta prevalencia de los síntomas musculoesqueléticos en cualquier región del cuerpo, y se asociaron principalmente con factores organizativos, como tipo de hospital, tipo de trabajo por turnos y frecuencia de cambios en el horario de trabajo, en vez de con factores personales.

De igual manera, en México los investigadores Montoya, Palucci, Do Carmo Cruz, y Taubert (2010) observaron que los dolores osteomusculares lumbares y cervicales; son los causantes de ausentismo laboral y poseen indicadores de morbilidad osteomuscular.

En tanto Guillén (2006), hizo mención en diversos estudios realizados en Europa y Estados Unidos, que mantener posturas corporales inadecuadas en los profesionales de enfermería genera tensión muscular que se traduce en dolor de cabeza, cuello y lumbalgias.

A nivel nacional el Instituto Nacional de Estadística e Informática (INEI, 2014) reportó que en los establecimientos de salud, el $48 \%$ de las enfermeras/ os entrevistadas/os manifestaron que siempre toman medidas de protección de riesgo laboral; el 33,4\% refirió que ocasionalmente su institución donde laboran les ha capacitado en la prevención de riesgos laborales o de bioseguridad y el 32,6\% manifestaron que casi siempre su institución le provee de medidas o equipos de protección. Además, en los accidentes laborales, el 14,9\% tuvo un accidente y el $8,4 \%$ dos accidentes, los trabajadores de la salud se exponen diariamente a múltiples riesgos ocupacionales.

La enfermera está enfocada al cuidado del paciente, pero a veces descuidan el riesgo que están expuestas, de manera que ciertos tipos de actividades y las condiciones ergonómicas inadecuadas, facilitan la aparición de riesgos. Entre los principales factores de riesgo para los trastornos musculoesqueléticos se incluyen: la organización del trabajo, los factores ambientales y la posible sobrecarga, una fuerza excesiva en la realización de determinadas tareas, la repetición de movimientos y posturas en el desarrollo de actividades laborales (De Souza et al., 2011).

Asimismo, el trabajo de investigación permitirá que las autoridades tomen medidas para la implementación de un programa de capacitación continua que identificará los riesgos, además sensibilizar a directivos y trabajadores/as sobre su existencia y forma de minimizarlos contribuyendo al mejoramiento de la práctica clínica. De igual manera, el instrumento aplicado, permitirá la aplicación para próximos estudios de investigación. Además encontrar la existencia de relación entre conocimientos, actitudes y prácticas en la prevención de los riesgos ergonómicos del profesional de Enfermería.

\section{MATERIALES Y MÉTODOS}

El estudio fue de enfoque cuantitativo, de diseño no experimental, de tipo correlacional y de corte transversal. Los instrumentos de conocimiento, actitudes y prácticas fueron elaborados por las autoras. Se realizó una prueba piloto con 60 profesionales de Enfermería que laboran en clínicas privadas, para medir la confiabilidad se procedió a realizar el coeficiente de Alfa de Cronbach, para las variables de actitudes, prácticas, y la prueba estadística de KR 20 para conocimiento. También se aplicó la validez de contenido mediante el juicio de expertos de 5 jueces, aplicando la $\mathrm{V}$ de aiken, obteniendo como resultado de 1 .

La muestra fue integrada por 130 profesionales de Enfermería, y la selección de la muestra fue realizada mediante el muestreo no probabilístico a conveniencia de las investigadoras. Se realizó la descripción de las variables utilizando la prueba estadística de chi cuadrado para relacionar una variable nominal y ordinal. 
Relación entre conocimientos, actitudes y prácticas en la prevención de los riesgos ergonómicos de los profesionales de enfermería de la Clínica Good Hope, Lima, 2016

\section{RESULTADOS}

Tabla 1

Relación entre conocimientos y actitudes en la prevención de los riesgos ergonómicos de los profesionales de enfermería que laboran en la Clínica Good Hope, Lima 2016.

\begin{tabular}{llll}
\hline Conocimientos & Negativa & \multicolumn{2}{c}{ Actitudes } \\
& 31 & 18 & Total \\
\hline \multirow{2}{*}{ Deficiente } & $23.80 \%$ & $13.80 \%$ & 49 \\
& 28 & 35 & $37.70 \%$ \\
Regular & $21.50 \%$ & $26.90 \%$ & 63 \\
& 12 & 6 & $48.50 \%$ \\
Bueno & $9.20 \%$ & $4.60 \%$ & 18 \\
& 34 & 66 & $13.80 \%$ \\
Total & $54.60 \%$ & $45.40 \%$ & 30 \\
& & & $100 \%$ \\
\hline
\end{tabular}

${ }^{*} \mathrm{X}^{2}=5,163 \mathrm{a} ; \mathrm{gl}=2 ; \mathrm{p}=0.076$

Se encontró que el $23.8 \%$ posee un conocimiento y actitud negativa contrario a un $4.6 \%$ que posee un conocimiento bueno con actitud positiva. Asimismo, se observa la prueba estadística de Chi cuadrado la cual evidencia un $p$ valor de 0,07 , siendo este ( $p>.05)$ de significancia, por lo que se acepta la hipótesis Ho y se rechaza la Ha. tal como se muestra en la tabla 1.

Tabla 2

Relación entre actitudes y prácticas en la prevención de los riesgos ergonómicos de los profesionales de Enfermería que laboran en la Clínica Good Hope, Lima 2016.

\begin{tabular}{lllll}
\hline Actitudes & Deficiente & Regular & Práctica & Total \\
\hline \multirow{2}{*}{ Negativa } & 22 & 37 & 12 & 71 \\
& $16,9 \%$ & $28,5 \%$ & $9,2 \%$ & $54.60 \%$ \\
Positiva & 12 & 29 & 18 & 59 \\
& $9.20 \%$ & $22.30 \%$ & $13.80 \%$ & $45.40 \%$ \\
Total & 34 & 66 & 30 & 130 \\
& $26.20 \%$ & $50.80 \%$ & $23.10 \%$ & $100 \%$ \\
\hline
\end{tabular}

$* \mathrm{X}^{2}=4,038 \mathrm{a} ; \mathrm{gl}=2 ; \mathrm{p}=0.133$

En la Tabla 2 se observa que del $100 \%$ de los encuestados, un $28.5 \%$ posee una práctica regular y una actitud negativa contrario a un $13.8 \%$ que posee una actitud positiva y una práctica buena. Además, se observa un $p$ valor de 0.133 , siendo este $(p>0.05)$ de significancia, Por tanto, se concluye que no existe relación significativa entre las variables de prácticas y actitudes de prevención de riesgos ergonómicos. 
Tabla 3

Relación entre conocimientos y prácticas en la prevención de los riesgos ergonómicos de los profesionales de enfermería que laboran en la Clínica Good Hope, Lima 2016.

\begin{tabular}{|c|c|c|c|c|}
\hline \multirow[b]{2}{*}{ Conocimiento } & \multicolumn{4}{|c|}{ Práctica } \\
\hline & Deficiente & Regular & Bueno & Total \\
\hline \multirow{2}{*}{ Deficiente } & 12 & 21 & 16 & 49 \\
\hline & $9.20 \%$ & $16.20 \%$ & $12.30 \%$ & $3 \%$ \\
\hline \multirow{2}{*}{ Regular } & 17 & 39 & 7 & 63 \\
\hline & $13.10 \%$ & $30.00 \%$ & $5.40 \%$ & $48.50 \%$ \\
\hline \multirow{2}{*}{ Bueno } & 5 & 6 & 7 & 18 \\
\hline & $3.80 \%$ & $4.60 \%$ & $5.40 \%$ & $13.80 \%$ \\
\hline \multirow{2}{*}{ Total } & 34 & 66 & 30 & 130 \\
\hline & $26.20 \%$ & $50.80 \%$ & $23.10 \%$ & $100 \%$ \\
\hline
\end{tabular}

$* \mathrm{X}^{2}=11,114 \mathrm{a} ; \mathrm{gl}=4 ; \mathrm{p}=0.025$

En la Tabla 3 se observa que del $100 \%$ de los encuestados, un $30 \%$ posee un conocimiento y práctica regular, un $9.2 \%$ posee un conocimiento y práctica deficiente. Asimismo, se observa la prueba estadística de Chi cuadrado lo cual evidencia un $\mathrm{p}$ valor de 0,025 , siendo este. Por tanto, se concluye que existe relación significativa y directa entre las variables de conocimientos y prácticas sobre riesgos ergonómicos.

\section{DISCUSIÓN}

En los últimos años se ha mostrado un interés particular por los riesgos de quienes laboran en servicios médicos y de salud; ya que componen un importante grupo laboral, que representa aproximadamente el $90 \%$ del recurso humano vinculado a instituciones hospitalarias. Este grupo, presenta condiciones particulares de trabajo, representadas por la continuidad de su servicio durante las 24 horas y las diferentes categorías de riesgo. Algunos estudios demuestran la existencia de riesgos físicos, químicos, biológicos, psicosociales e incompatibilidades ergonómicas, que de modo abierto o encubierto afectan a los profesionales que prestan servicios de salud (Tayupanta \& Ulco, 2012).

El personal de enfermería, desde el punto de vista epidemiológico, es considerado como un grupo especialmente vulnerable frente a los riesgos de su labor, hecho que se agrava muchas veces por la ausencia de cobertura específica en materia de salud laboral y por la carencia o no implementación de estándares de prevención a la exposición de riesgos que algunas veces podrían resultar fatales. Los problemas ergonómicos que se plantean relacionados al personal de enfermería están vinculados al uso de instrumentos médicos y de dispositivos de control, al proyecto de las instalaciones sanitarias y a la manipulación manual. Por otro lado, para la gran mayoría de los empleadores, los problemas ergonómicos no figuran entre los problemas principales en materia de salud y seguridad que deben resolver, pero cada vez es mayor el número de trabajadores a los que los afecta un diseño mal concebido (Briseño, Fernández, Herrera, y Enders, 2007)

Ruiz, Villavicencio, y Flores (2004), mencionaron que los profesionales de enfermería se encuentran expuestos a los accidentes laborales, principalmente por sobreesfuerzos, movimientos estresantes; resbalones, tropiezos y por objetos; además los turnos de tarde y de noche suelen contar con menos personal $\mathrm{y}$ frecuentemente con rendimientos laborales menos satisfactorios y adicionado a que las enfermeras tienen difícil acceso a transporte seguro y a comodidades básicas, motivos por el cual los riesgos ergonómicos se pueden acrecentar. Por lo tanto, afecta a la calidad de la atención de los servicios de enfermería.

Asimismo, señalaron que las enfermeras a menudo realizan actividades físicas de trabajo pesado como el levantamiento de cargas, posturas incómodas, transferencia de pacientes, operación de equipos riesgosos, etc. Además, la profesión enfermero está segunda en el ranking de carga de trabajo físico, después del trabajo industrial (Perez y Sánchez, 2009).

Los resultados encontrados en este trabajo de investigación revelan que el $48,5 \%$ de los profesionales 
de enfermería presentaron un conocimiento regular y el $37,7 \%$ un conocimiento deficiente en relación a la prevención de riesgos ergonómicos (figura 2). Del mismo modo, el 50,8 \% indicó una práctica regular y un $54,6 \%$ una actitud negativa (figura 3). Estos resultados son parecidos a lo reportado por Calderón (2014) en el Hospital Nacional Dos de Mayo; donde el $58 \%$ de los profesionales de enfermería indicó conocer sobre ergonomía, y el $42 \%$ no conoce.

Asimismo, Vega (2009) señaló que el desconocimiento de los principios o falta de aplicación de la mecánica corporal, lleva al personal de enfermería a estar permanentemente expuestos a la posibilidad de padecer lesiones por sobrecarga repetitivas. Asimismo, Briseño, Fernández, Herrera y Enders (2007), refirieron que hay mayor prevalencia de exposición de riesgos ergonómicos en personas que no toman las medidas correspondientes de control y que desconocen la forma que deben adoptar para evitar que el riesgo les cause daño (citado por Moya y Vinueza, 2013).

De acuerdo a lo indicado anteriormente, la OMS (2001) señaló que el aumento del conocimiento no asegura un cambio conductual, pero es importante, porque constituye el primer paso para el proceso del cambio.

Por otro lado, De Souza (2011) indicó que los accidentes laborales incrementan el absentismo, los permisos y el retiro por discapacidad. En efecto el estudio mostró que el 33,8\% de enfermeros manifestó haberse ausentado por motivos de salud.

En el año 2016 en la Clínica Good Hope hubo un $37.70 \%$ de profesionales de enfermería quienes solicitaron descanso médico, refiriendo tener lumbalgia. De igual manera Ruiz, Palomino, Gomero, y Llap, (2003) en Perú investigó la "Prevalencia, impacto en la productividad y costos totales de las principales enfermedades en el personal hospitalario" encontró que el absentismo representó el 29\% del tiempo total perdido por enfermedad y fueron por trastornos músculo esqueléticos con un promedio de 34.78 días de descanso médico. Jiménez (2014), refirió que cuando existe ausentismo se debe tener en cuenta los costos directos: el salario, la seguridad social, los días que le corresponde pagar a la empresa por la incapacidad. Y los costos indirectos: el tiempo que implica el buscar a la persona adecuada para sustituir al empleado, la formación que hay que darle a esa persona, la adaptación del nuevo trabajador al puesto de empleo y a la organización y a sus compañeros, la recarga de tareas que implica para el resto de los empleados, el impacto en la prestación del servicio hacia los pacientes.
Los hallazgos encontrados en el trabajo de investigación señalan que existe relación significativa entre los conocimientos y prácticas sobre las medidas de prevención de los riesgos ergonómicos en profesionales de enfermería con un p-valor de 0,025 (ver tabla 3). Además, los datos descriptivos revelan que el $30 \%$ de estos profesionales presentaron un conocimiento y práctica regular; en congruencia, la jefa de enfermeras en coordinación con la administración de la Clínica Good Hope, realizan programas de educación continua programados de manera sistemática, para mejorar los conocimientos de los profesionales de enfermería, y así dar calidad de atención pero la población no participa y no se preocupa en asistir.

Asimismo, otro estudio reportado por Manturano y Cabanillas, (2013) en su estudio ejecutado en Perú, mencionó que existe relación entre los conocimientos y prácticas sobre autocuidado en los profesionales de enfermería del servicio de Pediatría del HNGAI ( $\mathrm{p}$-valor $=0,009)$. Y sus resultados alcanzados fueron que un $42.2 \%$ de las enfermeras no conocen sobre autocuidado y muestra una práctica inadecuada sobre el mismo.

Esto coincide con lo manifestado por Arrivillaga y Salazar, (2005) quienes señalaron que un individuo puede conocer o desconocer la importancia de cualquier conducta de protección o de riesgo para la salud. Si la desconoce, notoriamente, las probabilidades de ejecución de una práctica saludable son menor que el desarrollo de una práctica poco o nada saludable. De igual manera Tobón afirmó que el conocimiento determina, en gran medida, la adopción de prácticas saludables, pues permiten a las personas tomar decisiones informadas y optar por prácticas saludables o de riesgo, frente al cuidado de su salud; sin embargo, estas determinaciones no dependen exclusivamente de la educación ni de la información, sino de factores internos y externos tales como los hábitos, actitudes, cultura, estilos de vida y la tecnología, entre otros (Manturano y Cabanillas, 2013).

Por otro lado, en el estudio de Segura y Ronquillo (2012) sobre "Factores de Riesgos Ergonómicos que inciden en la salud del personal de enfermería del área de Cuidados Intensivos" en Ecuador, demostró que los profesionales de enfermería no poseen conocimiento alguno sobre los factores de riesgos ergonómicos porque nunca antes habían sido capacitados en el tema y por ende no podían poner en práctica las medidas preventivas.

Ante lo referido, Molineros (2013) recomendó constantes capacitaciones sobre medidas preventivas 
en el personal de salud para minimizar las causas de riesgo de enfermedades ocupacionales, y crear conciencia en el cuidado de su salud. Además, se evidencia que la mayor parte de accidentes laborales o enfermedades profesionales son resultado de la interacción de múltiples factores de riesgo, entre ellos; falta de experiencia, la falta de personal, sobrecarga de trabajo, por desempeñar las actividades laborales en condiciones inseguras, porque no hay cultura de prevención, o no tienen los conocimientos necesarios, y carecen de elementos adecuados de protección personal.

En cuanto a la relación entre la presencia del dolor y las prácticas de prevención de riesgos ergonómicos, la investigación evidencia que hay asociación entres estas variables con un p-valor de 0,0004 (ver tabla 11), donde se puede resaltar que un $83,1 \%$ de encuestados presentaron algún tipo de dolor o molestia musculo esquelética en alguna parte de su cuerpo, siendo el $42,3 \%$ en la zona lumbar, $28.5 \%$ cervical y el $12,3 \%$ en las rodillas.

Montalvo, Cortés, y Rojas López (2015) encontraron resultados similares en un estudio realizado en Colombia, donde el personal de enfermería manifestó alguna molestia durante sus labores en los últimos 12 meses, siendo la espalda y el cuello los más afectados. La International Association for the Study of Pain (IASP, 2009) encontró que las aflicciones musculo esqueléticas son consecuencia del uso excesivo relacionado con el trabajo y el esfuerzo repetitivo, estas lesiones incluyen una variedad de trastornos que provocan dolor en los huesos, articulaciones, músculos o estructuras circundantes.

Además mencionó que las enfermeras permanecen en una postura fija por tiempo prolongado, adoptando posturas forzadas y les produce contracturas, así como procesos inflamatorios Calderón (2014). Se añade que las lumbalgias y dorsalgias se asocian con la "movilización de pacientes", "carga de elementos de más de $15 \mathrm{Kg}$ ", y "malas posturas al sentarse" (Briseño et al., 2007)

Existe un historial de molestias o lesiones de columna, propias del quehacer diario de enfermería. Generalmente no son tomadas en cuenta en los primeros años del ejercicio de la profesión, con el tiempo se produce un desgaste por varios factores, entre ellos, el paso de los años, sobrepeso, falta de tonicidad de músculos abdominales y la mala aplicación de la mecánica corporal, generando problemas a veces irreversibles en el estado físico (Mendoza, 2009).

Se observa en el estudio que hay relación entre el estado de salud y las prácticas para prevenir riesgos ergonómicos $(p=0.007)$ donde un $30,8 \%$ de los profesionales encuestados perciben que su estado de salud es bueno, además, presentan prácticas regulares de prevención de riesgos ergonómicos (ver tabla 12).

Otro estudio por Tayupanta (2008) argumentó que un $70 \%$ de los profesionales padecían de problemas ergonómicos por las inadecuadas prácticas ergonómicas.

Asimismo, Sánchez ( 2014) refirió que las enfermeras han enseñado a las personas a cuidarse, aunque ellas mismas no necesariamente cuiden su salud. Las enfermeras son afectadas por su entorno y su labor, una investigación muestra que el $60 \%$ de enfermeras tuvo prácticas de autocuidado inadecuadas, también conocimientos deficientes en cuidados de salud, baja autoestima y malestar emocional. De la misma manera, en el estudio de Garduño, Méndez, y Salgado (2012), se encontró que existe falta de compromiso en el profesional de enfermería frente al cuidado de su salud. Además, las enfermeras se acostumbran a convivir con la enfermedad por lo que poco hacen por cuidar su propia salud. Sin embargo, se puede sospechar que la mala práctica se deba a las inadecuadas condiciones laborales, en su medio ambiente de trabajo y está sometido a riesgos que generan problemas de salud, y frecuente ausentismo laboral (Segura y Ronquillo 2012).

De igual manera los datos descriptivos revelaron que el hecho de contar con conocimientos no condiciona una actitud positiva, lo que puede generar prácticas inapropiadas que potencian el riesgo de elevar la incidencia de esta problemática. Esto se apoya en el estudio realizado por Cabrera et al. (citado por González et al., 2013), quienes afirmaron que al indagar los conocimientos, son las actitudes las que determinan la manera en que se realizan e influencian las prácticas. Asimismo, Laza y Sánchez (2012), resaltaron que los conocimientos son necesarios para influir en la salud.

Por otro lado, Quintana, (2014) mencionó que son los enfermeros los primeros responsables de visibilizar y luchar por mejorar su calidad de vida en el trabajo, teniendo el compromiso de brindar un cuidado mejor.

De igual manera, Gil (2009) mencionó que las actitudes que desarrollan los profesionales están asociadas a los riesgos de su entorno laboral, siendo los riesgos de origen psicosocial de especial relevancia en el sector sanitario debido a que los profesionales trabajan en continua interacción con personas y normalizan esas actitudes. 
En cuanto a la relación de las actitudes y prácticas en la prevención de riesgos ergonómicos, no se encontró relación entre ambas variables con un p-valor de 0.133 (ver tabla 2).

Además, el 28,5\% de los profesionales de enfermería que manifestaron una actitud positivas presentaron prácticas regulares, en el estudio un $79,2 \%$ de los encuestados tienen la edad entre 25 a 54 años, perteneciente al grupo adulto intermedio (MINSA, 2010), la edad de los encuestados puede inferir en sus actitudes como lo demuestra Moya y Vinueza (2013) mencionó que un 90\%son adultos jóvenes, mientras que un $10 \%$ adultos mayores, se demostró que el adulto joven posee mayor fuerza física, conocimientos innovadores sobre la aplicación de la mecánica corporal, a diferencia de los adultos mayores que muestran desinterés de adoptar nuevas formas y técnicas en la movilización de pacientes, equipos, etc.; y prefieren seguir adoptando las mismas posiciones en la manipulación diaria, haciéndolos más sensibles a adquirir algún riesgo ergonómico: Las prácticas regulares pueden ser por temores como lo menciona De Souza et al., (2011) tener una enfermedad ocupacional que trae consigo el incremento de miedos y temores derivados de la inestabilidad profesional por posible destitución o separación del empleo, debida a los recurrentes ausentismos en las instituciones. Asimismo Almirall, Hernández, y Almirall, (2011) recomienda insistir en la necesidad del cambio de mentalidad hacia la seguridad y la prevención, es necesario incidir en que los conocimientos se traduzcan en actitudes, y esas actitudes en prácticas más seguras.

\section{Declaración de financiamiento y de conflicto de intereses:}

El estudio fue financiado por los autores, quienes declaran no tener algún tipo de conflicto de interés en la investigación realizada.

\section{Correspondencia:}

Paulina Raquel Tipantuña Malte

Licenciada en Enfermería. Clínica Americana Adventista Quito, Ecuador.

e-mail: paulinamalte@upeu.edu.pe

\section{REFERENCIAS BIBLIOGRÁFICAS}

Almirall, M., Hernández, D., \& Almirall, P. (2011). Conocimiento, riesgo y condiciones de trabajo en trabajadores de la atención primaria de salud en el municipio Playa. Retrieved January 10, 2017, from http://bvs.sld.cu/revistas/rst/vol12_3_11/rst01311.htm

Arrivillaga, M., \& Salazar, I. (2005). Creencias relacionadas con el estilo de vida de jóvenes latinoamericanos. Psicología Conductual, 13, 19-36. Retrieved from http:// proinapsa.uis.edu.co/redcups/Biblioteca/Documentos REDCUPS/Investigacion_Creencias_y_ Estilos_de vida.pdf

Briseño, C., Fernández, A., Herrera, R., \& Enders, J. (2007). Detección y evaluación de factores de riesgos laborales en el personal de enfermería del sector público. Revista de Salud Pública, 11(1), 42-54. Retrieved from https://revistas.unc.edu.ar/index.php/RSD/article/ viewFile/7239/8329

Calderón, G. (2014). Conocimiento sobre los riesgos laborales del profesional de enfermería en el Centro Quirúrgico del Hospital Nacional Dos de Mayo2014. Universidad Nacional Mayor de San Marcos. Retrieved from http://ateneo.unmsm.edu.pe/ateneo/ bitstream/123456789/4442/1/Calderon_Izaguirre_ Geovana_Karolina_2014.pdf

Creus, A. (2012). Técnicas para la prevención de riesgos laborales. (S. A. MARCOMBO, Ed.). Barcelona: LEXUS.

De Souza, C., Lima, J., Antunes, E., Schumacher, K., Moreira, R., \& De Almeida, T. (2011). Riesgos ergonómicos de lesión por esfuerzo repetitivo del personal de enfermería en el hospital. Enfermería Global, 10(23), 251-263. https://doi.org/10.4321/S1695-61412011000300018

Garduño, A., Méndez, V., \& Salgado, F. (2012). Autocuidado del Profesional de Enfermería de un Hospital Público, 19-27. Retrieved from http://www. uaemex.mx/revistahorizontes/docs/revistas/Vol5/2 AUTOCUIDADO.pdf

Gil-Monte, P. R. (2009). Editorial algunas razones para considerar los riesgos psicosociales en el trabajo y sus consecuencias en la salud pública. Rev Esp Salud Pública, 83(2), 169-173. Retrieved from http://www. msc.es/biblioPublic/publicaciones/recursos_propios/ resp/revista_cdrom/vol83/vol83_2/RS832C_169.pdf

González, D., Banguera, B., Gómez, L., \& Cruz, A. (2013). Artículo Corto Conocimientos , Actitudes y Prácticas sobre Prevención de Dolor Lumbar ., 3(1), 26-29.

Laza, C., \& Sánchez, G. (2012). Indagación desde los conocimientos, actitudes y prácticas en salud reproductiva femenina: algunos aportes desde la investigación ., 408-415. Retrieved from file://Users/ WendyReyes/Downloads/modelo-tipo-CAP (1).pdf

Manturano, Z., \& Cabanillas, M. (2013). Conocimiento y práctica de autocuidado del profesional de Enfermería del servicio de Pediatría del Hospital Nacional Guillermo Almenara Irigoyen , 35-42.

Melorose, J., Perroy, R., \& Careas, S. (2015). Prevención De Riesgos Ergonómicos. Confederación Regional 
de Organizaciones Empresariales de Murcia, 1, 1-11. https://doi.org/10.1017/CBO9781107415324.004

MINSA. (2010). Etapa de vida adulto. retrieved January 12, 2017, from http://www.tacna.minsa.gob.pe/uploads/ desp/Adulto_1S08.pdf

Molineros, M. (2013). Riesgo laboral del personal de salud del Hospital Nacional de Salud Mental de Guatemala, mayo - julio 2013. Universidad Rafael Landívar. Retrieved from http://recursosbiblio.url.edu. gt/tesiseortiz/2015/09/11/Molineros-Maria.pdf

Montalvo, A. A., Cortés, Y. M., \& Rojas López, M. C. (2015). Ergonomic Risk Associated To Musculoskeletal Symptoms in Nursing Staff. Hacia La Promoción de La Salud, 20(2), 132-146. https://doi.org/10.17151/ hpsal.2015.20.2.11

Moya, P., \& Vinueza, J. (2013). Riesgos Ergonómicos en el Personal de Enfermería que labora en los servicio de Medicina Interna, Emergencia, Cirugía/ Traumatología y Quirófano en el Hospital "San Luis de Otavalo" en el Periodo de Enero y Octubre del año 2013. Universidad Técnica del Norte. Retrieved from http://repositorio. utn.edu.ec/bitstream/123456789/2834/1/06 ENF 583 TESIS.pdf

OMS. (2001). Régimen alimentario, actividad física y salud. Retrieved January 9, 2017, from http://apps.who.int/gb/ archive/pdf_files/EB109/seb10914.pdf

Perez, S., \& Sánchez, P. (2009). "Riesgos Ergonómicos En Las Tareas De Manipulación De Pacientes, En Ayudantes De Enfermería Y Auxiliares Generales De Dos Unidades Del Hospital Clínico De La Universidad De Chile"., 58. Retrieved from http://repositorio.uchile.cl/tesis/ uchile/2009/me-perez_a/pdfAmont/me-perez_a.pdf

Quintana, M. (2014). Calidad de vida en el trabajo, personal de enfermería. Secretaría de Salud Pública, HermosilloMéxico. Universidad Concepción. Retrieved from http://repositorio.udec.cl/bitstream/handle/11594/1603/ Tesis_Calidad de Vida_en_el Trabajo.Image.Marked. pdf? - sequence $=1$
Ruiz, A., Villavicencio, M., \& Flores, M. (2004). \&quot; Factores de riesgo que intervienen en los accidentes laborales en el personal de enfermería. hospital fernando vélez PAÍZ.- MANAGUA, 2004.\&quot; UNIVERSIDAD NACIONAL AUTÓNOMA. Retrieved from http:// cedoc.cies.edu.ni/digitaliza/t339/portada.pdf

Ruiz, F., Palomino, J. C., Gomero, R., \& Llap, C. (2003). Prevalencia, impacto en la productividad y costos totales de las principales enfermedades en el personal hospitalario en un hospital al sur del perú en EL AÑO 2003. Retrieved from http://www.bvsde.paho.org/ bvsacd/cd27/prevalencia.pdf

Sánchez, B., Sámano, R., Chinchilla, D., \& Rodriguez, A. (2014). Autocuidado en enfermeras y su asociación con su estado emocional. Salud Pública de México, 56, 235236. Retrieved from http://www.scielosp.org/pdf/spm/ v56n3/v56n3a1.pdf

Segura, K., \& Ronquillo, A. (2012). Factores de Riesgos Ergonómicos que inciden en la salud del personal de enfermería del área de Cuidados Intensivos del Hospital Abel Gilbert Pontón de la Ciudad de Guayaquil, 2013. Universidad de Guayaquil. Retrieved from http:// repositorio.ug.edu.ec/bitstream/redug/8762/1/factores ergonómicos.pdf

Tayupanta, S., \& Ulco, C. (2012). Riesgos laborales en el Personal de Enfermería que labora en Sala de Operaciones del Hospital Carlos Andrade Marín, Quito, Junio, 2008. Universidad Central del Ecuador. Retrieved from http://www.dspace.uce.edu.ec/ bitstream/25000/620/1/T-UCE-0006-21.pdf

Vega, M. (2009). Mecánica corporal. Universidad del Aconcagua. Retrieved from http://bibliotecadigital.uda. edu.ar/objetos_digitales/117/tesis-3779-mecanica.pdf

Recibido: 08/02/2017 Aceptado: 05/05/2017 